\title{
Superconductivity from orbital nematic fluctuations
}

\author{
Hiroyuki Yamase ${ }^{1,2}$ and Roland Zeyher ${ }^{1}$ \\ ${ }^{1}$ Max-Planck-Institut für Festkörperforschung, Heisenbergstrasse 1, D-70569 Stuttgart, Germany \\ ${ }^{2}$ National Institute for Materials Science, Tsukuba 305-0047, Japan
}

(Dated: July 6, 2018)

\begin{abstract}
Recent experiments suggest that besides of antiferromagnetic fluctuations nematic fluctuations may contribute to the occurrence of superconductivity in iron pnictides. Motivated by this observation we study superconductivity from nematic fluctuations in a minimal two-band model. The employed band parameters are appropriate for iron pnictides and lead to four pockets for the Fermi line. It is shown that low-energy, long-wavelength nematic fluctuations within the pockets give rise to strong-coupling superconductivity whereas the large momenta density fluctuations between pockets are rather irrelevant. The obtained transition temperatures are similar to those typically found in the pnictides and are rather robust against repulsive Coulomb interactions. The superconducting and nematic states coexist in a large region of the phase diagram.
\end{abstract}

PACS numbers: 74.20.Mn, 75.25.Dk, 74.25.Dw, 74.70.Xa

Electronic analogues of nematic liquid crystals attract much interest and are observed in a number of condensed matter systems, such as quantum spins [1], twodimensional electron gases [2, 3], cuprates [4, 5], bilayer ruthenates [6], and iron pnictides [7]. In a nematic state the orientational symmetry is broken whereas the other symmetries are retained. Depending on electronic degrees of freedom responsible for nematic order, we may distinguish between three kinds of nematicity: charge 8 10], spin [11, 12], and orbital [13, 14] nematicity.

In the pnictides a nematic transition is observed just above the spin-density-wave (SDW) phase and is accompanied by a tetragonal-orthorhombic structural phase transition [7]. One thus expects both antiferromagnetic and nematic fluctuations near these transition lines. The nature of the nematic fluctuations may be either due to spins [15, 16] or due to orbitals 17 19]. Recent Raman scattering [20] experiments found direct evidence for low-frequency charge fluctuations [21, 22] in this region indicating that these nematic fluctuations involve orbital fluctuations even near the SDW phase. Furthermore, magnetic torque measurements [23] showed a nematic transition at higher temperatures which extends to a region where the SDW is already far away but where superconductivity still occurs.

The above experiments suggest an important role of nematic fluctuations for the superconductivity in the pnictides. A possible non-magnetic mechanism is also suggested by a recent discovery that some iron pnictides exhibit superconductivity with critical temperature $T_{c}$ $\sim 40-50 \mathrm{~K}$, which survives very far away from the SDW phase 24 26].

In this paper, we address the question whether orbital nematic fluctuations can give rise to high temperature superconductivity by employing a minimal two-band model for iron pnictides which exhibits four Fermi pockets and a nematic transition. We show that interpocket fluctuations which may violate Migdal's theorem and thus are difficult to calculate give only small contributions to $T_{c}$. On the other hand intrapocket fluctuations can be calculated reliably using the strong-coupling Eliashberg theory, which yields $T_{c}$ 's comparable to the observed ones in the pnictides. We obtain the superconductivity in a region moderately away from the nematic transition and also inside the nematic phase.

Our approach to superconductivity in pnictides is quite different from previous studies [27 30], although we share their views on the importance of orbital fluctuations. In Refs. 28 and 29 they considered the weak-coupling limit without quasi-particles renormalizations and obtained unrealistic high values for $T_{c}$ confined closely to the transition line of orbital order. Refs. 27 and 30 concluded that superconductivity is driven by short-wavelength orbital fluctuations and not by long-wavelength ones used by us.

Our model Hamiltonian has the form $H=H_{0}+H_{1}$ where the interaction part $H_{1}$ is given by

$$
H_{1}=\frac{g}{2} \sum_{i} n_{i-} n_{i-} .
$$

The difference density operator $n_{i-}$ is defined by $n_{i-}=$ $n_{i 1}-n_{i 2}$ with the density operator $n_{i \alpha}=\sum_{\sigma} c_{i \alpha \sigma}^{\dagger} c_{i \alpha \sigma}$. $i$ and $\sigma$ are site and spin indices, respectively, and $\alpha=$ 1,2 is a band index. $g$ is a coupling constant which is considered as a parameter in our model. An expression for $H_{0}$ suitable for pnictides is 31 ]

$$
H_{0}=\sum_{\mathbf{k}, \sigma, \alpha, \beta} \epsilon_{\mathbf{k}}^{\alpha \beta} c_{\mathbf{k} \alpha \sigma}^{\dagger} c_{\mathbf{k} \beta \sigma}
$$

with $\epsilon_{\mathbf{k}}^{11}=-2 t_{1} \cos k_{x}-2 t_{2} \cos k_{y}-4 t_{3} \cos k_{x} \cos k_{y}$, $\epsilon_{\mathbf{k}}^{22}=-2 t_{2} \cos k_{x}-2 t_{1} \cos k_{y}-4 t_{3} \cos k_{x} \cos k_{y}, \epsilon_{\mathbf{k}}^{12}=$ $-4 t_{4} \sin k_{x} \sin k_{y}$. Reasonable values for the hopping amplitudes are 31] $t=-t_{1}, t_{2} / t=1.5, t_{3} / t=-1.2, t_{4} / t=$ -0.95 , which we will also use in our calculations; the chemical potential is fixed to be $0.6 t$. In the following energies are always given in units of $t$. 
The above model shows for negative values of $g$ a nematic phase transition. Figure 1 depicts the result of a mean-field calculation of the phase diagram in the $T$ $g$ plane. Disregarding superconductivity the region to the left of the solid line is occupied by the normal state whereas to the right side of this line a nematic state is realized. The nematic state is homogenous, i.e., $\left\langle n_{i-}\right\rangle$ is independent of $i$, except for a small region bounded by the line with crosses and the dashed line in Fig. 1 where a modulated nematic state is found.

In the following we will study superconductivity from nematic fluctuations. We consider the usual Fock diagram for the electronic self-energy where the bosonic propagator describes nematic fluctuations. Since superconductivity is a Fermi surface effect, we may restrict the momenta to the region near the Fermi line of each pocket. In our case there are two hole pockets near the $\Gamma$ - and Mpoint and two electron pockets near the X- and Y-point. We denote them by $i=1 \ldots 4$, respectively, see the inset in Fig. 1. Assuming that the superconducting order parameter is constant on each individual pocket the Eliashberg equations for the gap $\Delta_{i}\left(i \omega_{n}\right)$ and the renormalization function $Z_{i}\left(i \omega_{n}\right)$ read as

$$
\begin{gathered}
\Delta_{i}\left(i \omega_{n}\right) Z_{i}\left(i \omega_{n}\right)=-\pi T \sum_{j, n^{\prime}} N_{j} \frac{\tilde{g}_{i j}\left(i \omega_{n}-i \omega_{n^{\prime}}\right)}{\left|\omega_{n^{\prime}}\right|} \Delta_{j}\left(i \omega_{n^{\prime}}\right), \\
Z_{i}\left(i \omega_{n}\right)=1-\pi T \sum_{j, n^{\prime}} N_{j} \frac{\omega_{n^{\prime}}}{\omega_{n}} \frac{\tilde{g}_{i j}\left(i \omega_{n}-i \omega_{n^{\prime}}\right)}{\left|\omega_{n^{\prime}}\right|} .
\end{gathered}
$$

$\omega_{n}$ is a fermionic Matsubara frequency and $N_{j}$ is the density of states of pocket $j$ at the Fermi energy. $\tilde{g}_{i j}\left(i \omega_{n}-\right.$ $\left.i \omega_{n^{\prime}}\right)$ is obtained from the microscopic pairing potential $W_{\alpha \beta}\left(\mathbf{k}, \mathbf{k}^{\prime}, i \nu_{n}\right)$ by averaging $\mathbf{k}$ and $\mathbf{k}^{\prime}$ independently over the Fermi lines of pockets $i$ and $j$, respectively, and by summing over $\alpha$ and $\beta$. $W$ is given by

$$
W_{\alpha \beta}\left(\mathbf{k}, \mathbf{k}^{\prime}, i \nu_{n}\right)=V_{\alpha \beta}^{2}\left(\mathbf{k}, \mathbf{k}^{\prime}\right) g\left(\mathbf{k}-\mathbf{k}^{\prime}, i \nu_{n}\right),
$$

where $\nu_{n}$ denotes a bosonic Matsubara frequency. $V$ represents the form factor

$$
V_{\alpha \beta}\left(\mathbf{k}, \mathbf{k}^{\prime}\right)=\sum_{\gamma, \delta} U_{\alpha \gamma}^{\dagger}(\mathbf{k})\left(\tau_{3}\right)_{\gamma \delta} U_{\delta \beta}\left(\mathbf{k}^{\prime}\right) .
$$

$U_{\alpha \beta}(\mathbf{k})$ is a unitary matrix which diagonalizes $H_{0}, \tau_{3}$ a Pauli matrix and $g\left(\mathbf{k}-\mathbf{k}^{\prime}, i \nu_{n}\right)$ is given by

$$
g\left(\mathbf{k}-\mathbf{k}^{\prime}, i \nu_{n}\right)=\frac{g^{2} \Pi\left(\mathbf{k}-\mathbf{k}^{\prime}, i \nu_{n}\right)}{1-g \Pi\left(\mathbf{k}-\mathbf{k}^{\prime}, i \nu_{n}\right)}+g_{0} .
$$

The first term in Eq. (77) is the retarded interaction mediated by nematic fluctuations, the second one is an instantaneous term accounting for repulsive Coulomb interactions. $\Pi$ stands for a single bubble of non-interacting nematic particle-hole excitations. The transition tem-

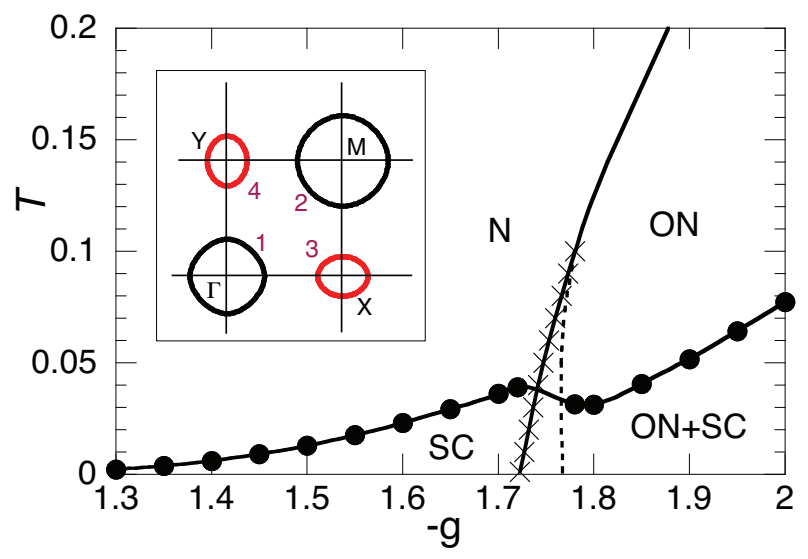

FIG. 1: (Color online) Phase diagram in the $T-g$ plane for $g_{0}=0$. The thick solid line separates the normal $(\mathrm{N})$ from the orbital nematic $(\mathrm{ON})$ phase which at low temperatures is modulated as indicated by the crosses. The dashed line is the instability line of the modulated to the homogeneous nematic state. The circles, smoothly joined by a thin solid line, separate the superconducting (SC) from the normal and nematic states. The inset shows the Fermi lines of the four pockets in the normal state.

perature $T_{c}$ to superconductivity is obtained from the condition that the largest eigenvalue of the matrix

$$
M\left(i, n ; j, n^{\prime}\right)=-\pi T N_{j} \tilde{g}_{i j}\left(i \omega_{n}-i \omega_{n^{\prime}}\right) /\left|\omega_{n^{\prime}}\right| / Z_{i}\left(i \omega_{n}\right)
$$

is equal to one.

The momentum-averaged pairing potentials $\tilde{g}_{i j}\left(i \nu_{n}\right)$ are important ingredients in the calculation of $T_{c}$. Figure 2 shows the dependence of $\tilde{g}_{i j}$ on the Matsubara frequency $\nu_{n}$, treated as a continuous variable, for various temperatures. The curves for $\tilde{g}_{44}\left(i \omega_{n}\right)$ of Fig. 2 (a) are always in the normal state. With decreasing temperature the correlation length of nematic fluctuations increases which implies that $\tilde{g}_{44}\left(i \omega_{n}\right)$ increases monotonically with decreasing temperature. At low temperatures $\tilde{g}_{44}\left(i \nu_{n}\right)$ assumes huge values at $\nu_{n}=0$ and then decays very fast with increasing frequency on an energy scale much smaller than $t$. In this frequency range $\tilde{g}_{44}\left(i \nu_{n}\right)$ varies only slowly with temperature which indicates that the attractive pairing interaction is rather insensitive to temperature and to critical fluctuations. For $g=-1.8$ [Fig. 2 (b)], the nematic instability occurs at the temperature $T_{n} \approx 0.125$. Here $\tilde{g}_{44}\left(i \nu_{n}\right)$ first increases monotonically with decreasing temperature until $T_{n}$ is reached. Entering the nematic state, $\tilde{g}_{44}\left(i \nu_{n}\right)$ is suppressed monotonically due to the development of nematic order. Except for this temperature dependence, $\tilde{g}_{44}\left(i \nu_{n}\right)$ is characterized by similar features as in Fig. 2 (a): large static values over a wide temperature region, a fast decay on an energy scale much smaller than $t$, and a weak temperature dependence at high frequencies.

The insets in Fig. 2 show the dependence of the non- 

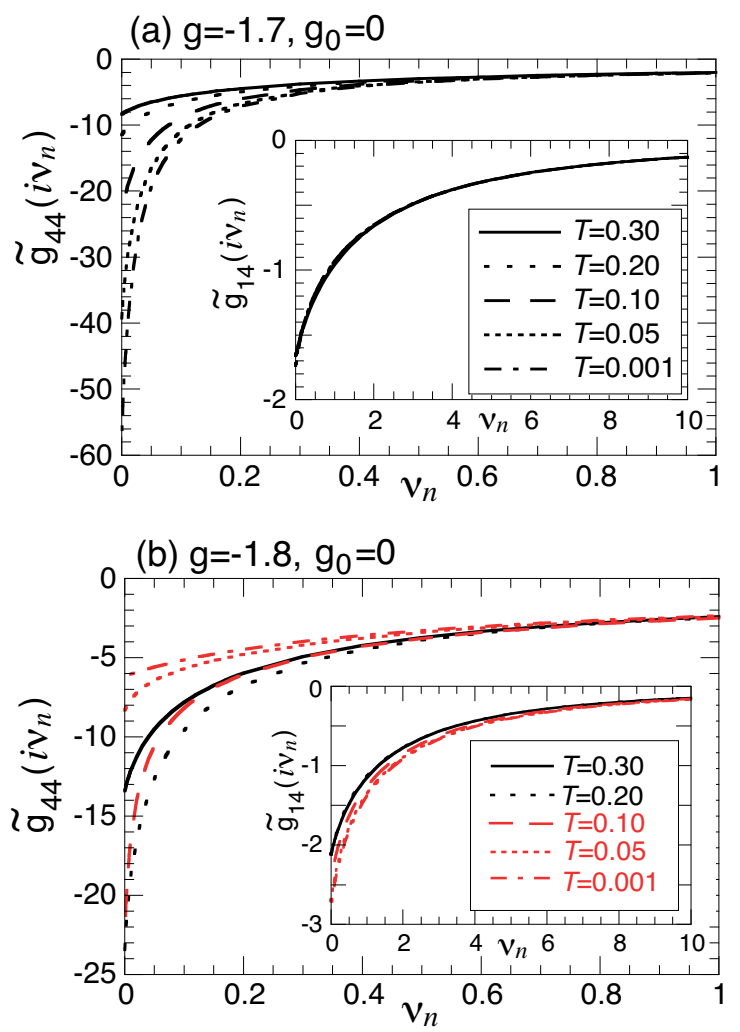

FIG. 2: (Color online) Retarded pairing interactions $\tilde{g}_{44}$ (main diagrams) and $\tilde{g}_{14}$ (insets) as a function of $\nu_{n}$ for different temperatures for $g=-1.7$ (a) and $g=-1.8$ (b). In the case (b) the nematic state occurs below $T=T_{n} \approx 0.125$.

diagonal interaction $\tilde{g}_{14}$ on $\nu_{n}$. In this case the momentum average involves large momenta in the effective interaction and the spectral function of the nematic fluctuations extends over a large frequency region of several $t$ 's. As a result the decay of $\tilde{g}_{14}$ with $\nu_{n}$ is much slower than in $\tilde{g}_{44}$ and characterized by an energy scale of $t$. On the other hand the low frequency values of $\tilde{g}_{14}$ are much smaller than those of $\tilde{g}_{44}$. In contrast to the case of $\tilde{g}_{44}$, the temperature dependence of $\tilde{g}_{14}$ is very weak. We also found that the curves for $\tilde{g}_{44}$ and $\tilde{g}_{14}$ in Fig. 2 are representative for all diagonal $i=j$ and non-diagonal $i \neq j$ interactions, respectively. The only exception is $\tilde{g}_{34}$ which becomes practically zero due to the matrix element $V_{\alpha \beta}\left(\mathbf{k}, \mathbf{k}^{\prime}\right)$ in Eq. (5).

We have solved Eqs. (3)-(4) numerically using up to 2546 Matsubara frequencies to get convergence. Figure 3 (a) shows the largest eigenvalue of the matrix $M\left(i, n ; j, n^{\prime}\right)$ as a function of temperature for $g_{0}=0$ and $g=-1.7$. Keeping only the diagonal 11,22,33,44 (non-diagonal 13,14,23,24 or 12,34) contributions in this matrix, one obtains the dotted (short-dashed or longdashed) curves; the solid line contains all contributions. The two curves corresponding to non-diagonal contributions are small down to low temperatures and thus do not lead to superconductivity. The closeness of the solid and
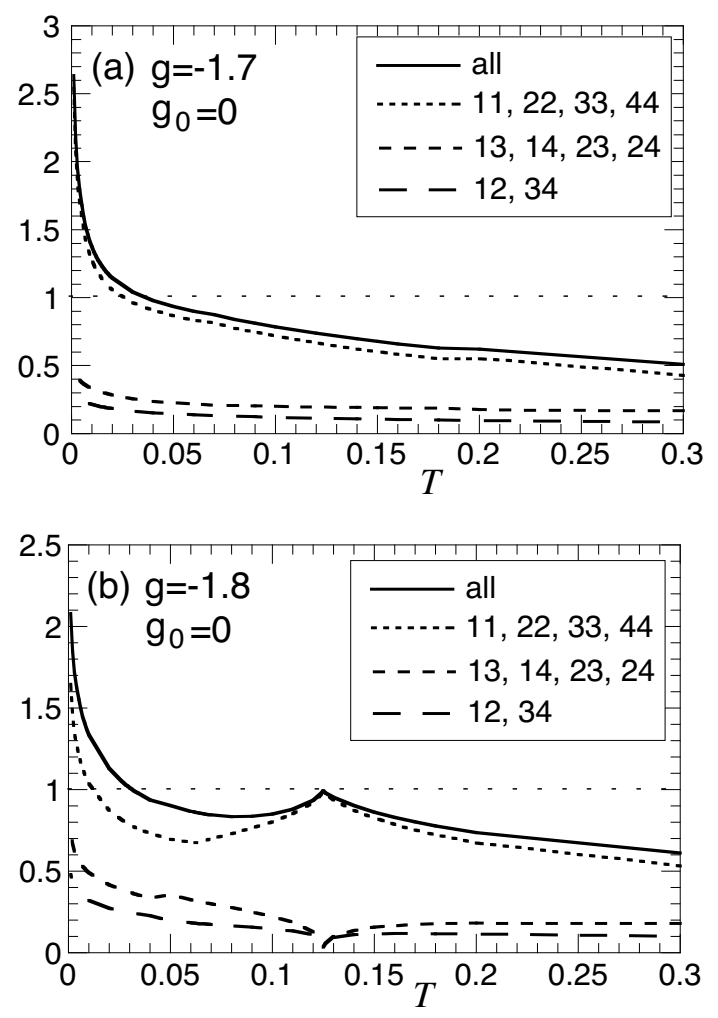

FIG. 3: Temperature dependence of the largest eigenvalue using only diagonal (dotted line), non-diagonal (short and long dashed lines) or all pairing interactions (solid line).

dotted lines proves that the total diagonal contribution is much larger than that of the non-diagonal terms. $T_{c}$ is thus determined to a large extent by excitations with small momentum transfer.

To understand the connection between $T_{c}$ and the frequency dependence of the pairing potentials $\tilde{g}_{i j}$ (Fig. 2), we first note that the zero frequency value of the pairing potentials with $i=j$ does not enter the largest eigenvalue when it crosses one. This follows immediately from Eqs. (3)-(4), highlighting the crucial role of $Z_{i}\left(i \omega_{n}\right)$. Thus the strong attractive part in the pairing potentials between 0 and $2 \pi T_{c}$ does not contribute at all to the instability towards superconductivity. At large temperatures mainly the flat high-frequency tail of $\tilde{g}_{i j}$ matters and already here the diagonal contribution accounts for most of the largest eigenvalue. Decreasing the temperature the low-frequency behavior of $\tilde{g}_{i j}$ becomes more and more important because of the substantial increase of the number of Matsubara frequencies contributing to the right-hand side of Eq. (3). This is the case also for Fig. 2 (b) although the low energy weight is suppressed at lower temperature in the nematic phase. As a result the huge increase of attraction towards low frequencies in the diagonal contributions dominates the total pairing potential, pushes the largest eigenvalue up to one and thus determines $T_{c}$. 
Figure 3 (b) shows the largest eigenvalue for $g=-1.8$ where the nematic phase is stable below $T_{n} \approx 0.125$. The non-diagonal contributions are again small and do not cross the value one. The diagonal and also the total contribution behave similar as in Fig. 3 (a) except in a temperature region around $T_{n}$ where the curves approach one from below. This findings follows directly from Eqs. (3)(44). At the transition point $T_{n}, \tilde{g}_{i i}\left(i \nu_{n}=0\right)$ and $Z_{i}\left(i \omega_{0}\right)$ tend to minus and plus infinity for $i=3$ and 4 , respectively; $\omega_{0}$ is the lowest fermionic Matsubara frequency. This implies that the largest eigenvalue approaches one from below, but never crosses one. This could be interpreted in terms of enhanced superconducting fluctuations near $T_{n}$. However, the assumptions underlying Eqs. (3)-(44) could break down near $T_{n}$, and non-Fermi liquid features might appear similar as in Ref. 32 .

The pairing potentials $\tilde{g}_{i j}\left(i \nu_{n}\right)$ are for $g_{0}=0$ attractive for all pocket indices $i, j$ and all Matsubara frequencies. As a consequence the components of the eigenvector $\Delta_{i}\left(i \omega_{n}\right)$ belonging to the largest eigenvalue have the same sign for all pockets and frequencies. This means that only the $s_{++}$symmetry for the order parameter can be realized. It is interesting that the second largest eigenvalue lies only a little below the solid line in Fig. 3 in the normal state. The corresponding eigenvector has $d$-wave symmetry, i.e., $\Delta_{1}\left(i \omega_{n}\right)=\Delta_{2}\left(i \omega_{n}\right)=0$ and $\Delta_{3}\left(i \omega_{n}\right)=-\Delta_{4}\left(i \omega_{n}\right)$ for all frequencies. To understand this approximate degeneracy, we first take into account only the rows and columns related to $\Delta_{3}$ and $\Delta_{4}$ in our matrix Eq. (8). Since $\tilde{g}_{34}$ is practically zero, $\Delta_{3}$ and $\Delta_{4}$ are essentially decoupled and there are two degenerate eigenvectors $\left(\Delta_{3}, \Delta_{4}\right) \propto(1,1)$ and $(1,-1)$, belonging to $s$ - and $d$-wave symmetry. Considering now also the rows and columns related to $\Delta_{1}$ and $\Delta_{2}$ the $d$-wave eigenvector does not change by symmetry whereas the $s$-wave eigenvector contains contributions from $\Delta_{1}$ and $\Delta_{2}$, with amplitudes comparable to $\Delta_{3}$ and $\Delta_{4}$, leading to our $s_{++}$state. The two eigenvalues are close to each other whereas the eigenvectors are quite different.

The filled circles in Fig. 1, smoothly joined by a thin solid line, show $T_{c}$ as a function of $g$. With increasing $-g, T_{c}$ monotonically increases because the nematic transition at $g_{n} \approx-1.72$ is approached and the nematic fluctuations increase. $T_{c}$ decreases somewhat when passing through the transition to the nematic state. Increasing $-g$ further enhances $T_{c}$ monotonically. Although the fluctuations are suppressed deep inside the nematic phase, they are sufficiently strong to drive a superconducting instability, leading to a coexistence of the superconducting and nematic states at lower temperatures. In the nematic phase, the Fermi pocket 3 (4) can disappear for $\left\langle n_{i-}\right\rangle>0(<0)$ at low temperatures, which is indeed the case for $-g \geq 1.8$. Experimentally, such a reconstruction has not been observed so far. However, $T_{c}$ is not affected much by such a Fermi surface reconstruction.

$Z_{i}\left(i \omega_{n}\right)$ assumes in general values much larger than

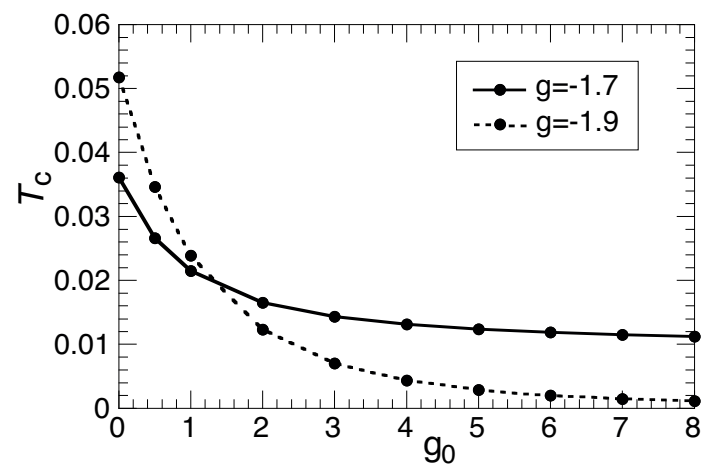

FIG. 4: Dependence of $T_{c}$ on the repulsive, instantaneous interaction $g_{0}$.

one at low frequencies, which indicates that the strongcoupling limit of superconductivity applies. Taking the free value $Z_{i}\left(i \omega_{n}\right)=1$ in Eq. (3) would lead in general to unphysically high critical temperatures and thus to incorrect conclusions. Approximating the frequency dependence of $\tilde{g}_{44}$ by a two-square-well model [33] the cutoff frequency would be about $\omega_{c} \sim 0.05$. Since on the average $T_{c} \sim 0.03$ we obtain the ratio $T_{c} / \omega_{c} \sim 0.6$ which is no longer small compared to one and thus again points to the presence of the strong-coupling limit. $T_{c}=0.03$ corresponds to about $50 \mathrm{~K}$ for the hopping $t=150 \mathrm{meV}$. Since $T_{c}$ is determined by intrapocket nematic fluctuations with an energy scale of $\omega_{c} \sim 0.05$, which is much smaller than characteristic electronic energies, Migdal's theorem should be well satisfied. This justifies our neglect of vertex corrections in Eqs. (3)-(4). If $\mathrm{T}_{c}$ would be dominated by interpocket fluctuations, for instance by $\tilde{g}_{14}$, the typical energy scale would be $\omega_{c} \sim 1$ according to Fig. 2 and Migdal's theorem could be violated.

In Fig. 4, we show the dependence of $T_{c}$ on the instantaneous repulsive interaction $g_{0}$, which will be present in real materials and might hinder nematic superconductivity. For the case $g=-1.7$ superconductivity evolves from the normal state and $T_{c}$ is only moderately suppressed by $g_{0}$. The components of the eigenvector belonging to the largest eigenvalue have at low frequency the same sign, hence $s_{++}$symmetry 34, but change the sign at a finite Matsubara frequency. This sign change leads to a partial cancellation of the repulsive terms in Eq. (3), which allows a non-vanishing solution for $\Delta_{i}\left(i \omega_{n}\right)$ even for a large $g_{0}$. Thus large repulsion terms become ineffective by retardation effects in our case, and not by nonconventional symmetries for the order parameter, such as $d$-wave. For the case of $g=-1.9$ in Fig. 4, superconductivity arises inside the nematic phase. Comparing the two panels in Fig. 2 indicates that retardation effects are in the nematic phase weaker than in the normal phase at low temperatures. The cancellation of strong repulsive terms in Eq. (3) by sign changes of the eigenvector is then impeded which explains the faster decay of $T_{c}$ and 
the vanishing of $T_{c}$ with increasing $g_{0}$.

In conclusion, we have considered a minimal twoband model for iron pnictides and found that orbital nematic fluctuations generate strong-coupling superconductivity with $T_{c}$ 's comparable to the observed ones in the pnictides. In contrast to a spin-fluctuation mechanism [35, 36], which is based on interpocket fluctuations, the present approach relies on intrapocket fluctuations which could yield a natural explanation for high- $T_{c}$ with a nodeless gap even in the case that only one pocket exists [37, 38]. Moreover, our non-magnetic mechanism is supported by the facts that long-wavelength charge fluctuations were observed by Raman scattering even close to the SDW phase 20], large $T_{c}$ 's occur in various pnictides even far away from the SDW phase $24-26$ and, at least in one case, near a nematic transition line 23]. We also found that it is absolutely necessary to include the renormalization of quasi-particles due to nematic fluctuations, which was neglected in the previous studies [28 30]. As a result $T_{c}$ is no longer tight closely to the nematic transition in agreement with the general phase diagram of pnictides [39] and superconductivity can occur also inside the nematic phase in agreement with the recent experiment 23].

The authors thank A. Eberlein and A. Greco for a critical reading of the manuscript and W. Metzner for discussions. H.Y. acknowledges support by the Alexander von Humboldt Foundation and a Grant-in-Aid for Scientific Research from Monkasho.

[1] K. Penc and A. M. Läuchli, in Introduction to Frustrated Magnetism, edited by C. Lacroix, P. Mendels, and F. Mila (Springer-Verlag, Berlin, 2011), p. 331.

[2] M. P. Lilly, K. B. Cooper, J. P. Eisenstein, L. N. Pfeiffer, and K. W. West, Phys. Rev. Lett. 82, 394 (1999).

[3] R. R. Du, D. C. Tsui, H. L. Stormer, L. N. Pfeiffer, K. W. Baldwin, K. W. West, Solid State Commun. 109, 389 (1999).

[4] S. A. Kivelson, I. P. Bindloss, E. Fradkin, V. Oganesyan, J. M. Tranquada, A. Kapitulnik, and C. Howald, Rev. Mod. Phys. 75, 1201 (2003).

[5] M. Vojta, Adv. Phys. 58, 699 (2009).

[6] A. P. Mackenzie, J. A. N. Bruin, R. A. Borzi, A. W. Rost, and S. A. Grigera, Physica C 481, 207 (2012).

[7] I. R. Fisher, L. Degiorgi, and Z. X. Shen, Rep. Prog. Phys. 74, 124506 (2011).

[8] S. A. Kivelson, E. Fradkin, and V. J. Emery, Nature (London) 393, 550 (1998).

[9] H. Yamase and H. Kohno, J. Phys. Soc. Jpn. 69, 332 (2000); 69, 2151 (2000).

[10] C. J. Halboth and W. Metzner, Phys. Rev. Lett. 85, 5162 (2000).

[11] A. F. Andreev and I. A. Grishchuk, Sov. Phys. JETP 60, 267 (1984).

[12] R. Fernandes and J. Schmalian, Supercond. Sci. Technol. 25, 084005 (2012).
[13] S. Raghu, A. Paramekanti, E-.A. Kim, R. A. Borzi, S. A. Grigera, A. P. Mackenzie, and S. A. Kivelson, Phys. Rev. B 79, 214402 (2009).

[14] W.-C. Lee and C. Wu, Phys. Rev. B 80, 104438 (2009).

[15] C. Fang, H. Yao, W.-F. Tsai, J. Hu, and S. A. Kivelson, Phys. Rev. B 77, 224509 (2008).

[16] C. Xu, Y. Qi, and S. Sachdev, Phys. Rev. B 78, 134507 (2008).

[17] F. Krüger, S. Kumar, J. Zaanen, and J. van den Brink, Phys. Rev. B 79, 054504 (2009).

[18] C.-C. Lee, W.-G. Yin, and W. Ku, Phys. Rev. Lett. 103, 267001 (2009).

[19] W. Lv, J. Wu, and P. Phillips, Phys. Rev. B 80, 224506 (2009).

[20] Y. Gallais, R. M. Fernandes, I. Paul, L. Chauvière, Y. -X. Yang, M. -A. Méasson, M. Cazayous, A. Sacuto, D. Colson, and A. Forget, arXiv: 1302.6255.

[21] H. Yamase and R. Zeyher, Phys. Rev. B 83, 115116 (2011).

[22] H. Yamase and R. Zeyher, arXiv: 1306.4267.

[23] S. Kasahara, H. J. Shi, K. Hashimoto, S. Tonegawa, Y. Mizukami, T. Shibauchi, K. Sugimoto, T. Fukuda, T. Terashima, A. H. Nevidomskyy, and Y. Matsuda, Nature (London) 486, 382 (2012).

[24] T. Hanna, Y. Muraba, S. Matsuishi, N. Igawa, K. Kodama, S.-i. Shamoto, and H. Hosono, Phys. Rev. B 84, 024521 (2011).

[25] S. Matsuishi, T. Hanna, Y. Muraba, S. W. Kim, J. E. Kim, M. Takata, S.-i. Shamoto, R. I. Smith, and H. Hosono, Phys. Rev. B 85, 014514 (2012).

[26] S. Iimura, S. Matsuishi, H. Sato, T. Hanna, Y. Muraba, S. W. Kim, J. E. Kim, M. Takata, and H. Hosono, Nat. Commun. 3, 943 (2012).

[27] T. D. Stanescu, V. Galitski, and S. Das Sarma, Phys. Rev. B 78, 195114 (2008).

[28] Y. Yanagi, Y. Yamakawa, N. Adachi, and Y. Ōno, J. Phys. Soc. Jpn. 79, 123707 (2010).

[29] H. Kontani and S. Onari, Phys. Rev. Lett. 104, 157001 (2010).

[30] H. Kontani, T. Saito, and S. Onari, Phys. Rev. B 84, 024528 (2011).

[31] Zi-Jian Yao, Jian-Xin Li, and Z. D. Wang, New J. Phys. 11, 025009 (2009).

[32] H. Yamase and W. Metzner, Phys. Rev. Lett. 108, 186405 (2012).

[33] J. P. Carbotte, Rev. Mod. Phys. 62, 1027 (1990).

[34] The symmetry changes to $s_{ \pm}$with $\Delta_{1,2}\left(i \omega_{0}\right)>0$ and $\Delta_{3,4}\left(i \omega_{0}\right)<0$ if $g_{0}$ is sufficiently large and $g>g_{n}$.

[35] I. I. Mazin, D. J. Singh, M. D. Johannes, and M. H. Du, Phys. Rev. Lett. 101, 057003 (2008).

[36] K. Kuroki, S. Onari, R. Arita, H. Usui, Y. Tanaka, H. Kontani, and H. Aoki, Phys. Rev. Lett. 101, 087004 (2008).

[37] Y. Zhang, L. X. Yang, M. Xu, Z. R. Ye, F. Chen, C. He, H. C. Xu, J. Jiang, B. P. Xie, J. J. Ying, X. F. Wang, X. H. Chen, J. P. Hu, M. Matsunami, S. Kimura, and D. L. Feng, Nat. Mater. 10, 273 (2011).

[38] S. He, J. He, W. Zhang, L. Zhao, D. Liu, X. Liu, D. Mou, Y.-B. Ou, Q.-Y. Wang, Z. Li, L. Wang, Y. Peng, Y. Liu, C. Chen, L. Yu, G. Liu, X. Dong, J. Zhang, C. Chen, Z. Xu, X. Chen, X. Ma, Q. Xue, and X. J. Zhou, Nat. Mater. 12, 605 (2013).

[39] G. R. Stewart, Rev. Mod. Phys. 83, 1589 (2011). 